\title{
Validation of a Malay Version of the Patient-Rated Wrist Hand Evaluation (PRWHE-MV)
}

\author{
Teh $\mathrm{KH}^{\mathrm{a}}$, Rosli MRI ${ }^{\mathrm{b}}$, Mohamed Saaid $\mathrm{F}^{\mathrm{a}}$, Arumugam $\mathrm{M}^{\mathrm{a}}$ \\ ${ }^{a}$ University Putra Malaysia, Pulau Pinang, Malaysia \\ ${ }^{\mathrm{b}}$ Hospital Pulau Pinang, Pulau Pinang, Malaysia
}

\begin{abstract}
INTRODUCTION: The Patient-Rated Wrist Hand Evaluation (PRWHE) is a widely used measure of patientreported disability, pain and appearance related to both hand and wrist disorders. It has been adapted crossculturally worldwide but not in Malaysia. We performed a translation and cross-cultural adaptation of the PRWHE into a Malay version (PRWHE-MV) with assessed reliability and validity in patients presented with hand and/or wrist disorders. MATERIAL AND METHODS: PRWHE-MV was developed via translation and resolution of cultural discrepancies. A total of 83 patients (38 men, 45 women) with the mean age of 38 (SD 14) years and hand and/or wrist disorders completed both PRWHE-MV and the Malay version of Quick DASH during their first orthopaedic hand clinic follow up, and a second PRWHE-MV at 48 hours later. Intraclass Correlation Coefficient (ICC) was used to assess test-retest reliability of the PRWHE-MV while Cronbach's alpha was calculated to assess the internal consistency. We tested the validity of PRWHE-MV against the Malay version of Quick DASH by calculating the nonparametric Spearman's correlation coefficient between these two measures. RESULTS: There was no major cultural problems during the forward and backward translation of PRWHE, except for a minor change owing to cultural discrepancy in toilet hygiene. The Cronbach alpha PRWHE-MV was $0.978(\mathrm{SEM}=5.5)$ at baseline and $0.979(\mathrm{SEM}=5.35)$ at the second assessment. For the test-retest reliability, ICC was 0.99. There was a strong direct correlation between the scores for the PRWHE-MV and the Quick DASH $(r=0.916, p<0.001)$. A majority of patients considered hand appearance somewhat important and were bothered in the past week. Their main concerns were gaining back normal hand function and subsequently returning to work. CONCLUSION: The PRWHE-MV showed high internal consistency, test-retest reliability and good validity in patients with hand and/or wrist disorders. We found the application and evaluation of the instrument to be feasible and understandable among patients in Malaysia.
\end{abstract}

KEYWORDS: Cross-cultural adaptation, Malay Translation, Patient-Rated Wrist Hand Evaluation.

\section{INTRODUCTION}

Patient self-report measures are essential and useful to facilitating clinicians in understanding their patients and monitoring their patients' disease progression better. Various upper extremity related patient self-evaluation measures have been developed, such as Michigan Hand

Corresponding Author:

Prof. Dr. Manohar Arumugam

Professor Orthopaedic,

Specialised in Hand and Microsurgery,

University Putra Malaysia.

Tel No: +6019-3694014

Email : manohar.arumugam@gmail.com
Outcome Questionnaire (MHQ), Disabilities of Arm, Shoulder and Hand Questionnaire (DASH), PatientRated Wrist Hand Evaluation (PRWHE), and PatientSpecific Functional Scale, Hand Injury Severity Scoring System (HISS). Each has its strengths and weaknesses.

Patient-Rated Wrist Hand Evaluation (PRWHE) is an eighteen item patient self-administered questionnaire consisting of three subscales which are designed to measure the pain and function of the wrist and hand joints. The subscales are pain subscale, function subscale and other concerns subscale. The pain subscale has five 
items regarding frequency and severity of pain, while the function subscale is subdivided into two subcategories: specific activities and usual activities. The specific activities subscale has six items while the usual activity subscale has four items. Each item is scored on a 0 to 10 scale. The total score is calculated by adding all the pain subscale scores and half of the sum of the function subscale score (sum of function subscale score divided by two). A score closer to 0 indicates less pain and a lower disability level while a score closer to 10 indicates more pain and disability. Other concerns subscale is a newly introduced subscale and it has three optional items on hand aesthetic. This subscale is not part of the scale scoring.

PRWHE has been translated into several languages worldwide. However, there is a lack of a Malay version PRWHE. The Malay language, being our national language, has been widely used in our daily lives. It is spoken, read and written in official settings in Malaysia. There is certainly a necessity to produce a Malay version of PRWHE in order to facilitate patients' correct understanding and responding to this self-evaluated form. A study concluded that the English form of the patients' self-evaluated measures should undergo translation and cultural adaptation beforehand prior to the assessment of people whose first language is not English ${ }^{1}$. In view of this, there is urgency for a Malay translated PRWHE which has undergone the test for good reliability and validity to be implemented in Malaysia as soon as possible.

It is definitely crucial to develop our own PRWHE-MV as it would contribute to medical field development. A well-developed PRWHE-MV will be a useful tool to help our clinicians in appraising the degree of hand and wrist related dysfunction of patients; to outline their treatment goals; to predict the prognosis of an illness; to evaluate the outcome of surgeries and to monitor patient disease progression. In addition, it can be used for the determination of the clinical status in the medicolegal assessment of hand and wrist related musculoskeletal injuries or act as a useful measurement for research purpose. Furthermore, a widely applied PRWHE-MV can assist local implant companies in enhancing and developing implant designs which would subsequently provide quality-controlled products to patients.
The main objective of our study is to translate and cross -culturally adapt the original PRWHE from English into a Malay version of the PRWHE according to the standard guidelines ${ }^{1,2}$. Once translated, the PRWHE-MV was then evaluated for its reliability and validity.

\section{MATERIALS AND METHODS}

We have obtained permission from the author of the original PRWHE, Dr Joy Christine MacDermid to translate and to cross-culturally adapt the PRWHE into a Malay version. Also, the study was conducted with prior approval from the Malaysian Medical Research and Ethics Committee (MREC). This study comprised two stages. The first stage involves the PRWHE translation and cross-cultural adaptation into a Malay version which was followed by the second stage of the validity and reliability testing of the PRWHE-MV.

\section{PRWHE translation and cross-cultural adaptation}

Our translation and cross-cultural adaptation of PRWHE was conducted following the international standard guidelines.1,2 This process comprised of five steps: forward translation, synthesis, back translations, expert committee review and pretesting.

\section{Forward translation}

Two native Malay speakers with no medical background translated the original PRWHE into Malay. Both translators are secondary school teachers with fluency in English. They were instructed to list possible translations to all difficult terms encountered and to translate them into words and sentences that can be appreciated effortlessly by a 12 year old Malaysian.

\section{Synthesis}

These two translations were then screened and integrated into a single Malay translated document by an expert committee consisting of nine professional healthcare workers: one Professor in Orthopaedic Hand and Microsurgery, three Rehabilitation Specialists and five hand therapists. 


\section{Back Translation}

Subsequently, three native English speakers who have never come across the original PRWHE beforehand translated the integrated Malay version back into English.

\section{Expert Committee Review}

The same expert committee counterchecked the back translation with the original PRWHE and studied the translation properly. Minor cultural adaptation was executed according to the Malaysian culture.

\section{Pre-test}

We ran pre-tests on 30 native Malay speakers with hand and/or wrist injuries. This pre-test was crucial to ensure there were no comprehension problems after translation.

\section{Participants}

\section{Sample size and participants}

The calculated sample size was 59 participants and was determined using the Sample Size Calculator v2.0.xls. We prospectively enrolled patients who visited the orthopaedic hand clinic from October 2019 till March 2020 at both Hospital Serdang and Hospital Pulau Pinang. The total number of participants were 106. With a $23(21.69 \%)$ drop-out rate, the final number of participants who completed this research was 83 participants.

\section{Inclusion criteria and exclusion criteria}

Inclusion criteria were the age of 18 years or older, the ability to fill out the questionnaire without great assistance, Malay as a first language and free of their immobilization apparatus prior to the assessment. Patients with concomitant injuries on other ipsilateral upper extremity joints, cognitive dysfunctions and neurologic diseases were excluded.

\section{Data collection}

At the first visit, all respondents' demographic characteristics and clinical diagnosis were documented (Table I). All participants were instructed clearly to fill out both the PRWHE-MV and the Quick DASH-Malay Version. Instead of the full-length DASH, we preferred the Quick DASH because it was shorter and thus, time saving. Participants returned 48 hours later at the clinic for a second assessment and to fill up the same Malay version of PRWHE. Alternatively, they were given a copy of the PRWHE-MV to fill up on the predetermined day (48 hours) and returned it during a subsequent clinic follow up later.

\section{Analysis of Reliability}

Test-retest reliability indicates the ability to replicate the same result in stable individuals. ${ }^{3}$ Participants filled out the PRWHE-MV two times within a 48 hour interval. We assumed their hand and wrist conditions remained stable during the assessment in these two different settings. We determined the test-retest reliability of the PRWHE-MV by calculating the Intraclass Correlation Coefficient (ICC) ${ }^{4}$. Fleiss JL et al. 5 interpreted and classified ICC values as: ICC less than 0.40 equals to poor reliability; ICC value in between 0.4 and 0.75 indicates moderate reliability; ICC value greater than 0.75 is considered excellent reliability.

\section{Analysis of Internal Consistency}

Internal consistency of the PRWHE-MV is the correlation between items and it implies whether several items within same category will produce similar scores ${ }^{3}$. We determined internal consistency of the PRWHE-MV by calculating Cronbach's alpha for all its subscales and total scores. ${ }^{6}$ Cronbach's alpha values ranging from 0.70 to 0.95 is deemed satisfactory. ${ }^{3}$

\section{Analysis of Validity}

We tested the validity of the PRWHE-MV against the Malay version of Quick DASH by calculating nonparametric Spearman's correlation coefficient. 
Table I Patients' Demographic and Clinical Characteristics

\begin{tabular}{|c|c|}
\hline Variables & Number (\%) \\
\hline Number of Participants & 83 \\
\hline \multicolumn{2}{|l|}{ Gender } \\
\hline Male & $38(45.78)$ \\
\hline Female & $45(54.21)$ \\
\hline \multicolumn{2}{|l|}{ Hand Dominance } \\
\hline Right & $78(93.97)$ \\
\hline Left & $5(6.02)$ \\
\hline \multicolumn{2}{|l|}{ Ethnicities } \\
\hline Malay & 77 (92.77) \\
\hline Indian & $4(4.82)$ \\
\hline Chinese & $2(2.40)$ \\
\hline \multicolumn{2}{|l|}{ Diagnosis } \\
\hline Distal End Radius Fracture & $16(19.27)$ \\
\hline Carpal Tunnel Syndrome & $13(15.66)$ \\
\hline Metacarpal Bone Fracture & $10(12.04)$ \\
\hline Trigger Fingers & $7(8.43)$ \\
\hline Scaphoid Fracture & $7(8.43)$ \\
\hline Tuft Fracture with Nail Bed Injury & $6(7.22)$ \\
\hline Wrist Ganglion Cyst & $5(6.02)$ \\
\hline Hand Laceration Wound+/-Tendon Cut & $5(6.02)$ \\
\hline Little Finger Volar Plate Contracture & $3(3.61)$ \\
\hline Dequervain Tenosynovitis & $3(3.61)$ \\
\hline Other & $8(9.64)$ \\
\hline
\end{tabular}

between these two measures. The interpretation is based on the Dancey and Reidy ${ }^{7}$ classification system, with the score more than 0.7 indicating strong correlation. We hypothesized that the PRWHE-MV will have an association with the Malay version of Quick DASH and the validity of the PRWHE-MV is sufficient if there is strong correlation between the total score of the PRWHE-MV and the Quick DASH-MV. We selected the Quick DASH questionnaire because it is the most established, acceptable and reliable upper extremity measure. ${ }^{8}$ Quick DASH has the advantage of being shorter and thus, time saving compared to the full length DASH questionnaire. Both tools have similar precision in upper extremity disorders and they are valid, reliable and responsive and can be used for clinical and/or research purposes. ${ }^{9}$ The DASH and Quick DASH have been translated and culturally adapted into the Malay language with proven reliability and validity among Malaysians with upper limb disorders. ${ }^{10}$

\section{RESULTS}

\section{Translation and cross-cultural adaptation}

We decided to culturally adapt one question in item 11 into "use tissue with affected hand" instead of "using bathroom tissue with affected hand" because most Malaysians are Muslim and thus, tissue is not the only cleaning method while using the bathroom. Also, 'bathroom tissue' can be confusing to Malaysians. On top of using bathroom tissues to clean after toileting, Malaysians utilize bathroom tissue for many other purposes in their lives.

\section{Reliability and validity analysis}

83 participants completed the study. All of them were able to read and understand the PRWHE-MV questions well and answer them effortlessly. 52 (62.65\%) were from Hospital Pulau Pinang, while 31(37.34\%) were from Hospital Serdang. The mean age $( \pm \mathrm{SD})$ of participants was $38.77( \pm 14.08)$ years with a range of 20 to 70 years old. Patients were predominantly female (54.21\%) and right-hand dominance (93.97\%).

The mean (SD) score for the total PRWHE-MV at baseline was 43.1(24.1) and 42.7(23.9) 48 hours late while the mean score for the Quick DASH-MV was 43.08 (24.09). Mean scores according to the PRWHE-MV subscales at baseline and at 48 hours are displayed in Table II. In the other concerns subscale, $74(89.2 \%)$ of patients considered hand appearance somewhat important; $8(9.6 \%)$ of them felt it was very important and only $1(1.2 \%)$ patient thought hand appearance was not important to him. On average, participants scored 6.04 (2.43) and agreed the appearance of their wrist/ hand bother them to a certain extent in the past week. 72 $(86.7 \%)$ of patients had other concerns at the end of questionnaire. Majority of them, 20(24.1\%) were worried whether they would gain back their normal hand function later. $19(22.9 \%)$ of them were concerned regarding their working status, while $11(13.3 \%)$ were keen to know the disease progression. Only $1(1.2 \%)$ patient worried about the permanent inability to lift weights with his injured hand and another 1 patient doubted the persistence of symptoms after operative intervention. 
Table II Mean Score of PRWHE-MV Baseline and at 48 hours, and Quick DASH-MV at baseline

\begin{tabular}{|c|c|c|}
\hline Scale & $\begin{array}{l}\text { Baseline } \\
\text { Mean (SD) }\end{array}$ & $\begin{array}{l}\text { At 48-hours } \\
\text { Mean (SD) }\end{array}$ \\
\hline \multicolumn{3}{|l|}{ PRWHE-MV } \\
\hline Total Score & $43.10(24.12)$ & $42.70(23.94)$ \\
\hline Pain Subscale & $21.92(12.42)$ & $21.58(12.29)$ \\
\hline Function Subscale & $21.18(13.49)$ & $21.08(13.45)$ \\
\hline \multicolumn{3}{|l|}{ Other concerns } \\
\hline \multicolumn{3}{|l|}{$\begin{array}{l}\text { How importance of the } \\
\text { appearance of the hand to } \\
\text { you? } \\
\text { n }(\%)\end{array}$} \\
\hline Very important & $8(9.6)$ & $8(9.6)$ \\
\hline Somewhat important & $74(89.2)$ & $74(89.2)$ \\
\hline Not Important & $1(1.2)$ & $1(1.2)$ \\
\hline $\begin{array}{l}\text { How much did the } \\
\text { appearance of your wrist/ } \\
\text { hand bother you in the } \\
\text { past week? (score 1-10) }\end{array}$ & $6.04(2.43)$ & $6.04(2.43)$ \\
\hline $\begin{array}{l}\text { Do you have any other } \\
\text { concerns? Yes, } \mathrm{n}(\%)\end{array}$ & $72(86.7)$ & $72(86.7)$ \\
\hline Cannot work & $19(22.9)$ & $19(22.9)$ \\
\hline Regaining normal function & $20(24.1)$ & $20(24.1)$ \\
\hline Persistent symptoms after operation & $1(1.2)$ & $1(1.2)$ \\
\hline Progressive worsening & $11(13.3)$ & $11(13.3)$ \\
\hline Unable to lift weights & $1(1.2)$ & $1(1.2)$ \\
\hline \multicolumn{3}{|l|}{ QUICK DASH-MV } \\
\hline Total Score & $43.08(24.09)$ & - \\
\hline
\end{tabular}

At baseline, the Cronbach alpha was $0.96(\mathrm{SEM}=2.36)$ for the pain, $0.98(\mathrm{SEM}=3.42)$ for the function and 0.98 $(\mathrm{SEM}=5.50)$ for the total scores. The corresponding values at 48 hours were 0.96 (SEM=2.61), 0.99 $(\mathrm{SEM}=3.18)$ and 0.98 (5.35), respectively (Table III).

Table III Cross-sectional precision and standard error of measurement of PRWHE-MV at baseline and 48 hours

\begin{tabular}{llll}
\hline \multicolumn{1}{c}{ PRWHE-MV } & Cronbach $\alpha$ & SD & SEM \\
\hline Baseline & & & \\
Pain Subscale & 0.964 & 12.419 & 2.35 \\
Function Subscale & 0.984 & 27.047 & 3.42 \\
Total Score & 0.978 & 37.082 & 5.50 \\
At 48 hours & & & \\
Pain Subscale & 0.955 & 12.285 & 2.61 \\
Function Subscale & 0.986 & 26.913 & 3.18 \\
Total Score & 0.979 & 36.903 & 5.35 \\
\hline
\end{tabular}

The test-retest reliability showed an ICC of 0.99 for the pain, 0.99 for the function and 0.99 for the total scores at the second evaluation indicating that the tools were perfectly consistent between baseline and 48 hours. (Table IV)
Table IV Interaction correlation coefficient

\begin{tabular}{lcc}
\hline PRWHE-MV & ICC & $95 \%$ CI \\
Pain Subscale & 0.99 & $0.99-1.00$ \\
Function Subscale & 0.99 & $0.99-1.00$ \\
Total Score & 0.99 & $0.99-1.00$ \\
\hline
\end{tabular}

\section{DISCUSSION}

It is absolutely important to establish our own PRWHEMV which will contribute to the medical field development. PRWHE-MV will be a useful tool to help our clinicians in understanding the extent of hand and wrist related dysfunctions in their patients; to outline their treatment goals; to predict the prognosis of illness; to evaluate the outcome of surgery and to monitor patient disease progression. In addition, it can be a medicolegal scale or a useful measurement for research purposes. Furthermore, a widely applied PRWHE-MV can assist local implant companies in their implant designs and subsequently provide quality controlled products to patients.

The result of this study has revealed that the PRWHEMV has good internal consistency and test-retest reliability. Moreover, the PRWHE-MV has strong direct correlation with the Quick DASH-MV. The translation and cross-cultural adaptation process used to obtain the PRWHE-MV followed the international guidelines applied to similar studies in other literatures. ${ }^{1,2}$ We decided to culturally adapt one question in item 11 into "use tissue with affected hand" instead of "using bathroom tissue with affected hand" because most Malaysians are Muslim and thus, tissue is not the only cleaning method while using bathroom. Also, 'bathroom tissue' can be confusing to Malaysians. On top of using bathroom tissue to clean after toileting, Malaysian do use bathroom tissue for many other purposes in their lives. This question was originally aimed to assess wrist flexion function. ${ }^{11}$ In fact, 'using tissue with affected hand' is testing the same function as 'using bathroom tissue', as both involve simultaneous active flexion of wrist joints, metacarpophalangeal joints and interphalangeal joints for cleaning purposes. Impaired wrist flexion function will pose similar difficulty for patients to withdraw tissue and effectively achieve cleaning purposes with the tissue at the same time. 
The PRWHE-MV has demonstrated a Cronbach alpha coefficient greater than 0.7 in both subscale scores and total score, which are absolutely adequate for this parameter..$^{10} \mathrm{~A}$ high Cronbach alpha coefficient indicate that our PRWHE-MV can provide better cross sectional precision for scores at the individual level. ${ }^{12}$ A similarly high internal consistency (Cronbach alpha values ranging 0.82 till 0.98 ) has been published in previous PRWHE cross cultural adaptation. ${ }^{13,14,15,16,17,18,19,20,21,22}$

There is no evidence available to aid in the selection of the time interval between questionnaire administration for a study of test-retest reliability for health status instruments. ${ }^{23} \mathrm{~A}$ study to compare two-time intervals (two days and two weeks) for test-retest reliability of health status instruments revealed no statistically significant differences. ${ }^{23}$ The lowest ICC score (0.81) was reported in the Hindi version of the PRWHE. ${ }^{21}$ According to the authors, it is likely that the participants in their study experienced an improvement in their status, which led to a lack of the stability needed for test-retest analysis. They agreed that two to seven days is a common retest interval and patients could be expected to remain stable. ${ }^{21}$ It is important that patients remain clinically stable during test-retest interval and short intervals between assessments are recommended to minimize the inaccuracy of secondary to clinical changes during this period. ${ }^{24}$ As such, the Arabic version of the PRWHE used a 30 minutes to 24 hours period for test-retest ${ }^{15}$ and the Brazilian version opted for two to seven days interval. ${ }^{18}$ Thus, a retest after two days of daily activities will minimize recall bias to patients and it can be assumed that with this shorter interval, patients tested remain clinically stable.

From this study, the PRWHE-MV and the Quick DASH-MV have demonstrated high correlation $(\mathrm{r}=$ 0.916) to each other. We have found that two similar studies established the validity of PRWHE against Quick DASH.22,25 Both PRWHE-MV and the Malay version of Quick DASH are expected to have strong correlations because both measures assess similar aspects (functions and pain) and their score are in the same direction (lower score indicate less disability). We have selected Quick DASH to construct validity, instead of general health assessment measures like Short Form-36 (SF-36) and EQ-5D questionnaire. This is because Quick DASH is a more region-specific measure pertaining to the upper limb and is definitely more sensitive and specific in detecting upper limb disabilities.

In recent years, hand aesthetic has been considered important and started gaining attention among patients with hand and wrist disorders. Thus, the latest PRWHE has added two hand aesthetic questions in the other concerns subscale. Our study documented that a majority of respondents, $74(89.2 \%)$ considered hand appearance as somewhat important, $8(9.6 \%)$ considered it as very important and only $1(1.2 \%)$ regarded it as not important. From our database, our respondents were of young age (mean age $=38.77$ ) and a slight majority of them were female $(54.21 \%)$. The only one respondent who belittled hand appearance was a 70-year-old gentleman. On average, we obtained a 6.04 score out of 10 from the question of 'how much did the appearance wrist/hand bother you in the past week'. Hand/wrist appearance has undoubtedly become a new aspect that clinicians should never overlook while treating patients with hand/wrist disease and is definitely an important aspect to be incorporated in hand/wrist evaluations.

The last question stated in the PRWHE-MV is 'do you have any other concerns'. $72(86.7 \%)$ of participants expressed their concerns by stating down their problems. A majority of them, $20(24.1 \%)$ wondered whether they would be able to regain their normal hand function in the future and $19(22.9 \%)$ were worried about whether they would be able to resume their working routine as before. Hand and wrist injuries undoubtedly impair hand function greatly especially if the dominant hand is involved. It will cost a great toll in the patient's quality of life and ability to resume working. Thus, this newly added question in the new PRWHE is helpful for clinicians to understand their patients better and formulate treatment goals for their patients accordingly.

A wide variety of clinical diagnosis in this study had the advantages of reducing the selection bias and provide higher generalizability. Shorter test-retest interval in our study ensured all patients were tested within a stable period, even in acute fracture cases. Thus, the ICC values were not affected despite diagnosis varying widely. Moreover, many similar studies in literatures 
have evaluated their reliability and validity based on diverse diagnosis. ${ }^{13,15,18,19,20}$

Malaysia is a country of multi-ethnic and multi-cultural backgrounds and thus, many languages can be found in our beloved country. However, Malay is the only recognized official language in this country. It is our national language and unifying language (Babasa Perpaduan). It is a well-known fact that Malay is a compulsory subject in both primary and secondary schools and a compulsory subject to pass in order to obtain the Malaysian Certificate of Education (Sijil Pelajaran Malaysia). Thus, all Malaysians learn Malay ever since schooling and master this language from young age. Studies showed that the basic level of Malay literacy rate among secondary school students in Malaysia is $95.2 \%{ }^{26}$ In view of this, the PRWHE-MV can address the needs of most Malaysians.

\section{CONCLUSION}

We translated and cross culturally adapted the original PRWHE to the Malay language according to the established international guidelines. The PRWHE-MV is well proven to be reliable and a valid measure for Malaysians.

\section{Financial disclosure and conflict of interest}

The authors of this manuscript certify that they have no affiliations with or involvement in any organization or entity with any financial interest in the subject matter or materials discussed in this manuscript.

\section{ACKNOWLEDGEMENTS}

It would not had been possible to complete this dissertation without the generous help from committee members consist of Professor Manohar Arumugam, Dr Mohd Izmi bin Ahmad @ Ibrahim, Dr Wan Rozita, Dr Zetty Noreeta, Noor Asfalina binti Abdullah, Nur Sunarti Pauzi, Salina Syed Sultan, Sonia Sharma A/P Ahok Kumar Sharma, and Phan Mei Fang. Not to forget our forward translators Faten binti Mohd Ibrahim, Ku Norhazaina $\mathrm{Ku}$ Adnan and backward translators Joel Kelly, Corinne Fraley, and Jared Douglas. We would like to thank Dr Sumaiyah Binti Mat (Statistician, University Malaya) for her kind advice on statistical analysis.

\section{REFERENCES}

1. Guillemin F, Bombardier C, Beaton D. Crosscultural adaptation of health-related quality of life measures: literature review and proposed guidelines. Journal of clinical epidemiology. 1993 Dec 1;46(12):1417-32.

2. Beaton DE, Bombardier C, Guillemin F, Ferraz MB. Guidelines for the process of cross-cultural adaptation of self-report measures. Spine. 2000 Dec 15;25(24):3186-91.

3. Knol DL. Measurement in medicine: a practical guide. Cambridge University Press; 2011.

4. Shrout PE, Fleiss JL. Intraclass correlations: uses in assessing rater reliability. Psychological bulletin. 1979 Mar;86(2):420.

5. Fleiss JL, Levin B, Paik MC. Statistical methods for rates and proportions. john wiley \& sons; 2013 Jun 12.

6. Cronbach LJ. Coefficient alpha and the internal structure of tests. psychometrika. 1951 Sep 1;16 (3):297-334.

7. Dancey CP, Reidy J. Statistics without maths for psychology. Pearson Education; 2007.

8. Szabo RM. Outcomes assessment in hand surgery: when are they meaningful. The Journal of hand surgery. 2001 Nov 1;26(6):993-1002.

9. Gummesson C, Ward MM, Atroshi I. The shortened disabilities of the arm, shoulder and hand questionnaire (Quick DASH): validity and reliability based on responses within the full-length DASH. BMC musculoskeletal disorders. 2006 Dec;7(1):44.

10. Al-Husuny A, Rampal L, Arumugam M, Rahman HB, Zakaria JB. Validation of a Malay Version of Disability of Arm, Shoulder and Hand Questionnaire (MVDASH).

11. MacDermid JC. The PRWE/PRWHE update. J Hand Ther. 2019 Apr-Jun;32(2):292-294.

12. Kennedy CA. The DASH and Quick DASH outcome measure user's manual. Institute for Work \& Health; 2011.

13. Wah JW, Wang MK, Ping CL. Construct validity of the Chinese version of the patient-rated wrist evaluation questionnaire (PRWE-Hong Kong Version). Journal of Hand Therapy. 2006 Jan 1;19 (1):18-27. 
14. Fairplay T, Atzei A, Corradi M, Luchetti R, Cozzolino R, Schoenhuber R. Cross-cultural adaptation and validation of the Italian version of the patient-rated wrist/hand evaluation questionnaire. J Hand Surg Eur Vol. 2012;37 (9):863-70.

15. Hasani FN, MacDermid JC, Tang A, Kho ME. Cross-cultural adaptation and psychometric testing of the Arabic version of the Patient-Rated Wrist Hand Evaluation (PRWHE-A) in Saudi Arabia. Journal of Hand Therapy. 2015 Oct 1;28(4):41220.

16. Kim JK, Kang JS. Evaluation of the Korean version of the patient-rated wrist evaluation. Journal of Hand Therapy. 2013 Jul 1;26(3):238-44.

17. Hemelaers L, Angst F, Drerup S, Simmen BR, Wood-Dauphinee S. Reliability and validity of the German version of "the Patient-rated Wrist Evaluation (PRWE)" as an outcome measure of wrist pain and disability in patients with acute distal radius fractures. Journal of Hand Therapy. 2008 Oct 1;21(4):366-76.

18. Da Silva Rodrigues EK, Fonseca MD, MacDermid JC. Brazilian version of the Patient Rated Wrist Evaluation (PRWE-BR): Cross-cultural adaptation, internal consistency, test-retest reliability and construct validity. Journal of Hand Therapy. 2015 Jan 1;28(1):69-76.

19. Ozturk O, Sari Z, Ozgul B, Tasyikan L. Validity and reliability of the turkish "patient-rated wrist evaluation" questionnaire. Acta Orthop Traumatol Turc. 2015 Mar 1;49(2):120-5.

20. Imaeda T, Uchiyama S, Wada T, Okinaga S, Sawaizumi T, Omokawa S, Momose T, Moritomo H, Gotani H, Abe Y, Nishida J. Reliability, validity, and responsiveness of the Japanese version of the Patient-Rated Wrist Evaluation. Journal of Orthopaedic Science. 2010 Jul 1;15(4):509-17.

21. Mehta SP, Mhatre B, MacDermid JC, Mehta A. Cross-cultural adaptation and psychometric testing of the Hindi version of the patient-rated wrist evaluation. Journal of Hand Therapy. 2012 Jan 1;25(1):65-78.

22. Rosales RS, García-Gutierrez R, Reboso-Morales L, Atroshi I. The Spanish version of the PatientRated Wrist Evaluation outcome measure: crosscultural adaptation process, reliability, measurement error and construct validity. Health and quality of life outcomes. 2017 Dec;15(1):169.

23. Marx RG, Menezes A, Horovitz L, Jones EC, Warren RF. A comparison of two time intervals for test-retest reliability of health status instruments. Journal of clinical epidemiology. 2003 Aug 1;56 (8):730-5.

24. De Vet HC, Terwee CB, Mokkink LB, Knol DL. Measurement in medicine: a practical guide. Cambridge University Press; 2011 Aug 11.

25. Sandelin H, Jalanko T, Huhtala H, Lassila H, Haapala J, Helkamaa T. Translation and validation of the Finnish version of the patient-rated wrist evaluation

26. Chew FP. Literacy among the secondary schools' students in Malaysia. International Journal of Social Science and Humanity. 2012 Nov 1;2(6):546. 